Curr Opin Clin Nutr Metab Care. 2017 May ; 20(3): 227-231. doi:10.1097/MCO.0000000000000369.

\title{
Timing of the initiation of parenteral nutrition in critically ill children
}

\author{
Lissette Jimenez, M.D. ${ }^{1}$, Nilesh M. Mehta, M.D. ${ }^{2}$, and Christopher Duggan, M.D., MPH ${ }^{1}$ \\ ${ }^{1}$ Division of Gastroenterology, Hepatology and Nutrition, Boston Children's Hospital, Harvard \\ Medical School, Boston, MA \\ 2Division of Critical Care Medicine, Department of Anesthesiology, Perioperative and Pain \\ Medicine, Boston Children's Hospital, Harvard Medical School, Boston, MA
}

\begin{abstract}
Purpose of Review-To review the current literature evaluating clinical outcomes of early and delayed parenteral nutrition initiation among critically ill children.

Recent Findings-Nutritional management remains an important aspect of care among the critically ill, with enteral nutrition (EN) generally preferred. However, inability to advance enteral feeds to caloric goals and contraindications to EN often leads to reliance on parenteral nutrition (PN). The timing of PN initiation is varied among critically ill children, and derives from an assessment of nutritional status, energy requirements, and physiologic differences between adults and children, including higher nutrient needs and lower body reserves. A recent randomized control study among critically ill children suggests improved clinical outcomes with postponing initiation of PN to 1 week after admission to the pediatric intensive care unit (PICU).
\end{abstract}

Summary-Although there is no consensus on the optimal timing of PN initiation among critically ill children, recent literature does not support the immediate initiation of PN on PICU admission. A common theme in the reviewed literature highlights the importance of accurate assessment of nutritional status and energy expenditure in deciding when to initiate PN. As with all medical interventions, the initiation of PN should be considered in light of the known benefits of judiciously provided nutritional support with the known risks of artificial, parenteral feeding.

\section{Keywords}

Parenteral nutrition; intensive care unit; children

\section{Introduction}

Despite more than 40 years of PN use in children, there remains no clear consensus on the optimal timing of its initiation among children who are critically ill. Nutrition optimization

Address correspondence to: Christopher Duggan, MD. MPH, Center for Nutrition, Division of Gastroenterology, Hepatology and Nutrition, Boston Children's Hospital, 333 Longwood Ave., Boston, MA, 02115, Phone: 617.355.7612,

christopher.duggan@ childrens.harvard.edu.

Conflicts of Interest

None. 
remains an important aspect of care among critically ill children in order to prevent microand macronutrient deficiencies, avoid negative nitrogen balance, and to encourage anabolism during a fluctuating metabolic state. However, concerns about the harmful effects of PN have prompted a risk benefit assessment when utilizing this mode to achieve nutrient delivery in the critically ill patient.

Guidelines for critically ill adults by the American Society of Parenteral and Enteral Nutrition (ASPEN) suggest clinical correlation and evaluation on an individual case basis. The strength of recommendations in this area is limited due to multiple studies with varied statistical significance, patient populations with wide-ranging nutritional deficits, and differences in illness severity. Studies in critically ill adults have suggested a potential clinical outcome benefit among patients with delayed initiation of PN supplementation by 1 week (1). The clinical benefits of early or late PN are less well known among critically ill children. Recently a large randomized controlled trial examined the benefits of early (on day 1 after PICU admission) vs. delayed PN (on Day 8) among critically ill children. The results of this study suggest benefits in clinical outcome in the delayed PN arm (2). Findings of this study mirror those found in critically ill adults and question the potential benefits of immediate PN initiation (within 24 hours of admission) among critically ill children.

\section{Current guidelines for parenteral nutrition supplementation}

The 2016 guidelines from ASPEN and Society of Critical Care Medicine (SCCM) for nutritional supplementation among critically ill adults recommends assessing nutritional status and measuring energy expenditure by indirect calorimetry (when available for determination of reliable caloric goals) upon admission. Enteral feeds are recommended to begin within 48 hours of admission to an intensive care unit (ICU) and early initiation of PN suggested in cases where there is an anticipated disruption to enteral nutrition, or contraindication. In patients without malnutrition or those with a low risk for malnutrition, PN introduction may be delayed for a week (3). Recommendations for delayed initiation of PN are based on studies that showed favorable outcomes among late PN initiation compared to early PN (1). In a large multicenter RCT study among critically ill adults, patients with delayed PN initiation for one week had a shorter hospital stay, reduced health costs, and were less likely to acquire new ICU infections compared to those with PN initiation within 48 hours of ICU admission. Alternatively, a smaller trial conducted at 2 sites, randomized critically ill adults to either continued EN or EN plus PN supplementation in patients who were unable to reach at least $60 \%$ of their energy goals (measured by indirect calorimetry) on day 3 of ICU admission. Although there were no differences in mortality or length of stay between the two groups, the group receiving supplemental PN had a 30\% reduction in nosocomial infection risk and more antibiotic-free days (4). The extrapolation of the results in these studies to the wider adult critical care population is limited due to differences in severity of illness, nutritional status of patients, and wide-ranging statistical significance. Based on the observed study limitations, recommendations on the timing of PN supplementation among critically ill adults are suggested to be applied on an individual case basis $(3,5)$. 
With respect to the timing of parenteral support in children, it is important to realize that infants and children have unique physiologic and metabolic needs that make them different than adults (6). These include a substantially higher metabolic requirement for energy, protein and micronutrients (per kg body weight) compared with adults, as well as relatively lower reserves of fat and protein. The combination of these factors, plus a high prevalence of prematurity and other factors that predispose children to malnutrition, generally mean that the threshold to begin enteral or parenteral nutrition support in hospitalized children is lower than among adults.

Nonetheless, formal guidelines about the precise timing of PN initiation in children are generally lacking from the literature. The ASPEN clinical guidelines on nutrition support in critically ill children recommends screening to evaluate nutritional status, measuring energy expenditure with the use of indirect calorimetry if available (standard equations are often unreliable), and initiation of EN as a preferred route of nutrition provision. There is no clear consensus on the optimal timing for PN as a supplement to insufficient EN (7). General pediatric guidelines advise initiation of $\mathrm{PN}$ when minimal enteral nutrition is anticipated longer than 3-5 days in children with malnutrition or low birth weight, and at 5-7 days among well-nourished children $(8,9)$.

\section{Evaluation of energy deficits and timing of parenteral supplementation}

The interplay between energy needs and changes in metabolism during severe illness, and fluctuations in endogenous energy may influence the optimal timing of PN supplementation in adults $(10,11)$. This change in metabolism during critical illness is mediated by hormones that orchestrate a state of catabolism to meet the nutrient requirements for survival during a period of decreased nutritional intake and is hypothesized to possibly influence energy requirements (10). However, in the modern era, overfeeding may occur in the early phase of severe illness due to unintended excess of exogenous sources of energy in the setting of decreased energy expenditure, and has been correlated with poor outcomes (12). Secondary effects observed in cases of overfeeding include hyperglycemia, hyperlipidemia, increased infection risks, and liver steatosis $(10,11)$. On the other hand, failure to provide adequate energy and protein during acute critical illness has been associated with poor outcomes in the PICU population. These studies demonstrated the benefits of early enteral nutrient administration and there were no benefits of early parenteral administration of nutrients (13, 14). All in all, accurately evaluating the energy expenditure among the critically ill and applying it to the patient's shifting metabolic demands are essential to determine the goal caloric needs, and should play a role in planning optimal timing of PN (Figure 1 - from ref $10)$.

Taking advantage of the metabolic stress response and being cautious for possible overfeeding in the first few days of illness may improve overall outcomes of early parenteral nutrition supplementation in adults $(10,11)$.

Among children, literature in recent years suggests little or no hypermetabolism and possibly hypometabolism after a variety of insults (15-17). Furthermore, estimating equations for REE are likely to be inaccurate and can both overestimate and underestimate 
true energy needs $(3,15,16)$. Studies examining the risks and benefits of PN in critical illness must take into account the true energy needs of individual patients.

\section{Clinical outcomes of early and delayed parenteral nutrition in critically ill children}

To date, there is one available trial that has evaluated clinical outcomes of the timing of parenteral nutrition initiation among critically ill children. Fivez et al. recently conducted a multicenter randomized control trial among critically ill children, comparing early (day 1 of ICU admission) or late (after 7 days of admission) initiation of PN (2). Primary clinical outcomes aimed to compare the incidence of new acquired infections and length of ICU stay. Other clinical outcomes included mortality, duration of mechanical ventilation support, renal replacement therapy, and serum markers of liver dysfunction.

The trial included 1440 patients with ages ranging from term newborns to 17 years of age. Critically ill patients who were anticipated to have an ICU stay greater than 24 hours and who were judged to be at least moderately at risk of malnutrition (as defined by the screening tool for risk of nutritional status and Growth (STRONGkids)) were included in the study. Randomization was stratified by age and diagnosis. Critically ill children were assigned to either early (within 24 hours) or late PN (after 1 week) in addition to enteral nutrition that was started in the first days of admission. PN was continued in both groups until enteral feeds reached $80 \%$ of the estimated overall caloric goal. Of note, the composition of PN, advancement rate of EN, estimated caloric goals, and glycemic control protocols varied across site locations based on institutional guidelines.

Outcomes observed in the delayed PN group were uniformly more favorable than in the group that received early parenteral nutrition. Critically ill children receiving late PN supplementation were observed to have a lower rate of new infections (10.7\% compared to $18.5 \%, \mathrm{p}<0.001$ ), with blood stream and upper airway infections decreasing by $50 \%$ compared to the early PN group. ICU length of stay was reduced by an average of 2.7 days ( $\mathrm{p}<.002)$ and there was an average 4.1 day reduction in total length of stay ( $\mathrm{p}<.01)$ among patients who received delayed PN initiation. No differences were observed in 30-day mortality rates between the two groups. Other secondary outcome measures demonstrated a reduction in mechanical ventilation support time and decreased risk for requiring renal replacement therapy, and a reduction in liver dysfunction as measured by liver function tests. Interestingly, children who were among the late PN group developed a higher rate of hypoglycemic events and had higher C-reactive protein (CRP) plasma levels ( $\mathrm{p}<.007)$ compared to the early PN group. As discussed by Fivez et al., it is unclear how the potential risk of increased episodes of hypoglycemia or the elevated inflammatory marker of CRP generally contributes to the observed outcome risks and benefits among those who received delayed parental nutrition. Overall the findings of this randomized trial suggest improved clinical outcomes among critically ill children who have a delay in parenteral nutrition supplementation. The external validation of the results of this trial has been questioned due to a number of limitations and design aspects. 


\section{Early versus late parenteral nutrition in critically ill children. Areas of considerations in study design}

There are several areas to consider when interpreting the study by Fivez, et al., including the methods of determining energy goals, the evaluation of malnutrition, and the overall study design wherein children received PN as early as the day of ICU admission. In the accompanying editorial to the paper, the trial was noted to have high internal validity, but limited external validity (18). Factors contributing to a reduction in generalizability included the utilization of STRONGkids as a screening tool that has not been validated to accurately assess the degree of malnutrition among critically ill children, and may not correctly reflect the degree of malnutrition of patients included in the study $(5,18,19)$. In the trial, the median BMI Z score was -0.5 and the $25^{\text {th }} \%$ was -1.5 , suggesting that there was not a robust representation of children with severe malnutrition. Beyond the use of STRONGkids as a malnutrition assessment tool and report of BMI, there were no other measures obtained (such as arm anthropometrics or laboratory indicators of nutritional status) to assess the degree of malnutrition in the cohort studied. In a recent study, the STRONGKids and other similar screening methods did not adequately identify children with malnutrition who were otherwise recognized as malnourished through standard anthropometric measures (20). This aspect of the trial is relevant as it challenges the claim that a delayed PN strategy may be beneficial in vulnerable children with severe malnutrition or high malnutrition risk. Indeed, the finding that immediate initiation of PN in well-nourished children is associated with adverse outcomes is essentially supportive of current practice to limit PN initiation to 3-5 days in undernourished children and 5-7 days in well-nourished children $(8,9)$.

Furthermore, the use of equations to estimate total caloric goals, although practical, may not reflect accurate metabolic demands in the critically ill. Estimation of energy expenditure, as opposed to measuring metabolic needs with indirect calorimetry, could falsely over or under estimate caloric goals $(16,21)$. Overfeeding in PN, which typically occurs in the early phases of illness, could potentially contribute to unfavorable outcomes among those who received early PN initiation (18, 22). Similar criticism was considered in the 1990's when routine pre-operative PN was delivered to malnourished adult patients, with observations of adverse outcomes in mildly malnourished patients thought to be secondary to an overfeeding and/or hyperglycemia effect rather than use of PN per se (23).

In addition, one might also question the premise of the Fivez et al., trial, namely that it is standard of care for PN to begin on day 1 of PICU admission. We and others generally defer the start of PN until other forms of nutrition support have proven unsuccessful, and that the duration of inadequate enteral nutrition at least last 5 days. In the trial, the mean LOS in the ICU was approximately 8 days and fewer than $5 \%$ of subjects were on ECMO, suggesting that more subjects received PN in the trial than would have actually received it outside of the study's inclusion criteria. Finally, more than $77 \%$ of patients randomized to the late PN arm were discharged from the ICU before Day 7 (18). Therefore, a majority of the late PN group did not receive any PN supplementation.

Our current guidelines for parental nutrition initiation rely on risk stratification of malnutrition and our ability to accurately assess energy expenditure via indirect calorimetry 
$(3,16)$. The results of the trial support the avoidance of PN initiation on admission to the PICU. However, in patients with pre-existing malnutrition, or an inability to achieve enteral nutritional caloric goals, earlier administration of PN (i.e., within 3-5 days of ICU admission) is likely still warranted (5).

\section{Conclusion}

Nutritional status and total energy expenditure play a significant role in determining the need for PN. There are no current standards in measuring the degree of malnutrition among critically ill children, and accurately estimating energy expenditure is essential in deciding the need for PN supplementation. The study by Fivez et al. has provided useful insights into the role of supplemental PN in critically ill children, however studies using a validated and reproducible measure of malnutrition are warranted before its results can be extrapolated to all at risk children. The finding that initiation of PN on day 1 is associated with adverse outcomes is supportive of current practice to limit PN initiation to 3-5 days in undernourished children and 5-7 days in well-nourished children. Complex cases warrant individual consideration.

\section{Acknowledgments}

None.

Financial support and sponsorship

Funding: K24DK104676 (CD), P30DK040561 (CD)

\section{References and recommended reading}

Papers of particular interest, published within the annual period of review, have been highlighted as:

*Of special interest

**Of outstanding interest

1. Casaer MP, Mesotten D, Hermans G, Wouters PJ, Schetz M, Meyfroidt G, et al. Early versus late parenteral nutrition in critically ill adults. The New England journal of medicine. 2011; 365(6):50617. [PubMed: 21714640]

**2. Fivez T, Kerklaan D, Mesotten D, Verbruggen S, Wouters PJ, Vanhorebeek I, et al. Early versus Late Parenteral Nutrition in Critically Ill Children. The New England journal of medicine. 2016; 374(12):1111-22. First RCT in critically ill children evaluating the clinical outcomes of early and late parenteral nutrition. Critically ill children who had PN delayed for one week had improved outcomes in rate of acquisition of new infections and length of stay in the ICU compared to early PN administration. [PubMed: 26975590]

*3. McClave SA, Taylor BE, Martindale RG, Warren MM, Johnson DR, Braunschweig C, et al. Guidelines for the Provision and Assessment of Nutrition Support Therapy in the Adult Critically Ill Patient: Society of Critical Care Medicine (SCCM) and American Society for Parenteral and Enteral Nutrition (A.S.P.E.N.). JPEN Journal of parenteral and enteral nutrition. 2016; 40(2): 159-211. Guidelines for early and delayed parenteral nutrition initiation in critically ill adults. Patients who are at high nutrition risk or serverely malnoursihed may benefit from early PN intiaition following ICU admission when enteral nutrition is not anticipated to advance or is contraindicated. Patients who are at low nutrition risk or mildly malnourished are suggested to start PN after 7 days if not meeting caloric goals with enteral feeds. [PubMed: 26773077] 
4. Heidegger CP, Berger MM, Graf S, Zingg W, Darmon P, Costanza MC, et al. Optimisation of energy provision with supplemental parenteral nutrition in critically ill patients: a randomised controlled clinical trial. Lancet (London, England). 2013; 381(9864):385-93.

*5. Balaguer M, Jordan I. Time of parenteral nutrition in paediatric critical care patients, prior nutritional status probably makes the difference? Journal of thoracic disease. 2016; 8(8):1869-71. Editorial commentary on RCT study by Fivez et al. that addressess potential limitations to study. [PubMed: 27619337]

6. Cunningham JJ. Body composition and nutrition support in pediatrics: what to defend and how soon to begin. Nutrition in clinical practice : official publication of the American Society for Parenteral and Enteral Nutrition. 1995; 10(5):177-82. [PubMed: 8552010]

7. Mehta NM. Compher C. A.S.P.E.N. Clinical Guidelines: nutrition support of the critically ill child. JPEN Journal of parenteral and enteral nutrition. 2009; 33(3):260-76. [PubMed: 19398612]

8. Collier, S., Gura, K., DeLoid, L., Dalton, M., Summers, L. Parenteral Nutrition. In: Sonneville, K., Duggan, C., editors. Manual of Pediatric Nutrition. Fifth. Shelton, Connecticut: People's Medical Publishing House; 2014. p. 196-248.

9. Hartman, C., Riskin, A., Shamir, R. Parenteral Nutrition. In: Duggan, C.Watkins, JB.Koletzko, B., Walker, WA., editors. Nutrition in Pediatrics 2. Fifth. Shelton, CT: People's Medical Publishing House (PMPH); 2016. p. 1035-90.

*10. Oshima T, Heidegger CP, Pichard C. Supplemental Parenteral Nutrition Is the Key to Prevent Energy Deficits in Critically Ill Patients. Nutrition in clinical practice : official publication of the American Society for Parenteral and Enteral Nutrition. 2016; 31(4):432-7. Review article that focuses on metabolic demands during severe illness, and timing of parenteral nutrition to minimize adverse effects of potential overfeeding in adults. [PubMed: 27256992]

11. Oshima T, Hiesmayr M, Pichard C. Parenteral nutrition in the ICU setting: need for a shift in utilization. Current opinion in clinical nutrition and metabolic care. 2016; 19(2):144-50. [PubMed: 26828579]

12. Mehta NM, Bechard LJ, Dolan M, Ariagno K, Jiang H, Duggan C. Energy imbalance and the risk of overfeeding in critically ill children. Pediatric critical care medicine : a journal of the Society of Critical Care Medicine and the World Federation of Pediatric Intensive and Critical Care Societies. 2011; 12(4):398-405.

13. Mehta NM, Bechard LJ, Zurakowski D, Duggan CP, Heyland DK. Adequate enteral protein intake is inversely associated with 60-d mortality in critically ill children: a multicenter, prospective, cohort study. The American journal of clinical nutrition. 2015; 102(1):199-206. [PubMed: 25971721]

14. Mehta NM, Bechard LJ, Cahill N, Wang M, Day A, Duggan CP, et al. Nutritional practices and their relationship to clinical outcomes in critically ill children--an international multicenter cohort study*. Critical care medicine. 2012; 40(7):2204-11. [PubMed: 22564954]

15. Mehta NM, Costello JM, Bechard LJ, Johnson VM, Zurakowski D, McGowan FX, et al. Resting energy expenditure after Fontan surgery in children with single-ventricle heart defects. JPEN Journal of parenteral and enteral nutrition. 2012; 36(6):685-92. [PubMed: 22539159]

16. Mehta NM, Bechard LJ, Leavitt K, Duggan C. Cumulative energy imbalance in the pediatric intensive care unit: role of targeted indirect calorimetry. JPEN Journal of parenteral and enteral nutrition. 2009; 33(3):336-44. [PubMed: 19126761]

17. Mehta NM, Duggan CP. Nutritional deficiencies during critical illness. Pediatric clinics of North America. 2009; 56(5):1143-60. [PubMed: 19931068]

*18. Mehta NM. Parenteral Nutrition in Critically Ill Children. New England Journal of Medicine. 2016; 374(12):1190-2. Editorial commentary on RCT study by Fivez et al. that addressess potential limitations to study. [PubMed: 26975731]

19. Spagnuolo MI, Liguoro I, Chiatto F, Mambretti D, Guarino A. Application of a score system to evaluate the risk of malnutrition in a multiple hospital setting. Italian journal of pediatrics. 2013; 39:81. [PubMed: 24373709]

20. Chourdakis M, Hecht C, Gerasimidis K, Joosten KF, Karagiozoglou-Lampoudi T, Koetse HA, et al. Malnutrition risk in hospitalized children: use of 3 screening tools in a large European 
population. The American journal of clinical nutrition. 2016; 103(5):1301-10. [PubMed: 27099244]

21. Zusman O, Theilla M, Cohen J, Kagan I, Bendavid I, Singer P. Resting energy expenditure, calorie and protein consumption in critically ill patients: a retrospective cohort study. Critical care. 2016; 20(1):367. [PubMed: 27832823]

*22. Li Y, Huang Y. Early versus Late Parenteral Nutrition in Critically Ill Children. The New England journal of medicine. 2016; 375(4):384-5. Editorial commentary on RCT study by Fivez et al that addressess potential limitations to study.

23. Perioperative total parenteral nutrition in surgical patients. The Veterans Affairs Total Parenteral Nutrition Cooperative Study Group. The New England journal of medicine. 1991; 325(8):525-32. [PubMed: 1906987] 


\section{Key Points}

- Similarly to observations in adult studies, improved clinical outcomes were generally observed among critically ill children with delayed initiation of parenteral nutrition in the ICU.

- While PN on first day of PICU admission cannot be generally recommended, the precise timing of PN initiation needs to be individualized.

- The optimal timing of PN as a supplement to EN remains unclear in children with severe malnutrition and high risk of nutritional deterioration.

- Close monitoring for overfeeding while on parenteral nutrition among the critically ill is warranted, given poor correlation of estimated calculation for energy expenditure.

- $\quad$ Accurate assessment of energy expenditure and degree of malnutrition will play a large role in deciding appropriate timing of PN supplementation. 


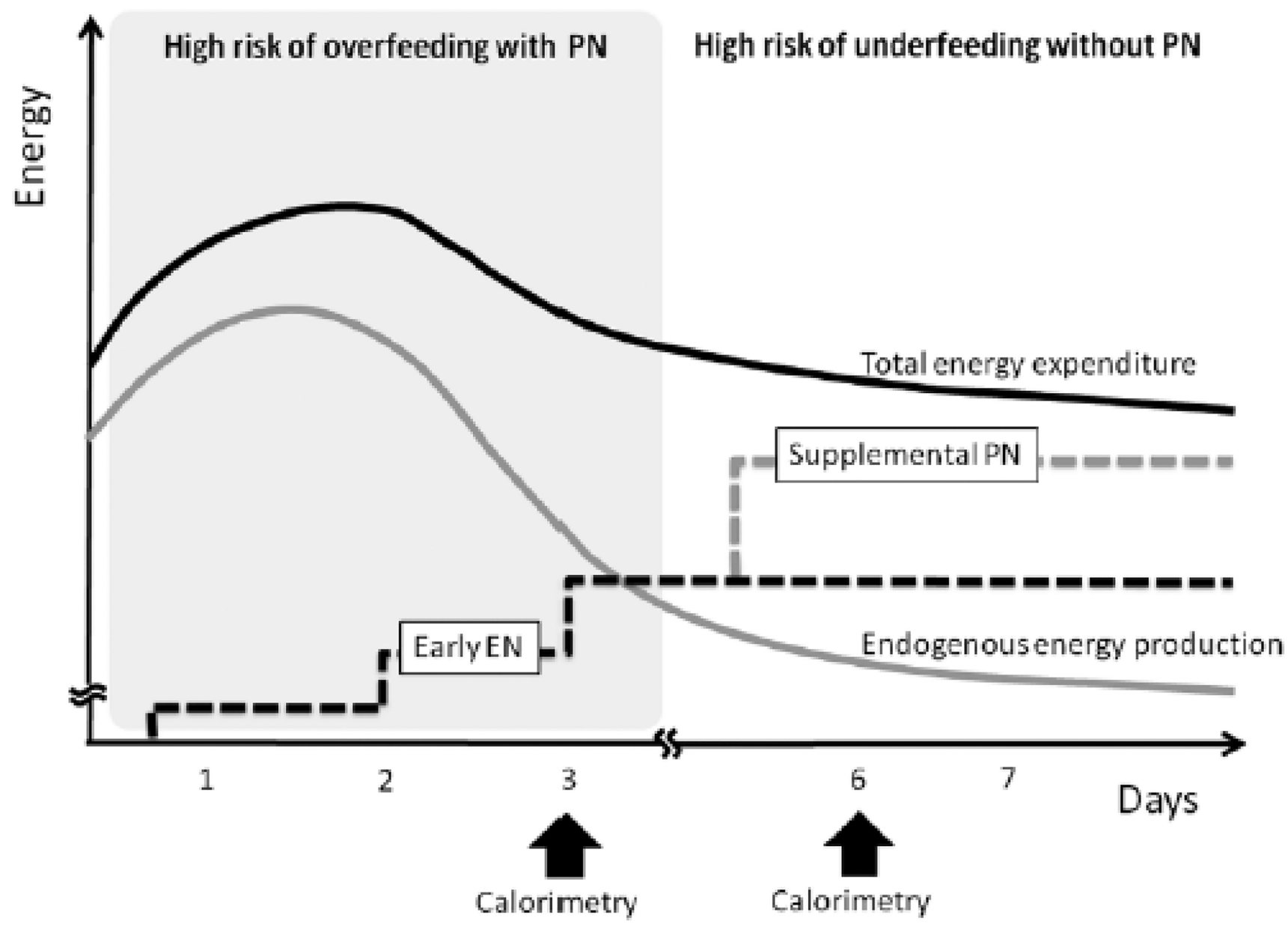

Figure 1.

Proposed optimal combination of enteral nutrition and parental nutrition may help avoid overfeeding and underfeeding. Reprinted with permission from Ref. (10). 